33 Crothers, T. D.: Diseases of inebriety.

therenger, S. V.: Treatment of the insane. Journal of American Medical Association, Oct. 24, 1896.

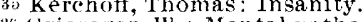

3e Gieseger, W.: Mental pathology and therapeutics.

rinnesos session

38 Bouchard, Chas.: Auto-intoxication.

39 Aulde, John. Sew York Nedical Journal, Aug, 15, 1896.

* Blech, J. M : Mathews Medical Quarterly, October, 1896.

4) Mackirlane, J. W.: Insomnia and its therapentics.

t2 Buchnill and Tuke: Psychologic medicine.

43 Alienist and Neurologist, October, 1895.

44 MeFarland, R. H.: Relation of operative gynecology to insanity, Lancet Chinic, A ug. 29, 1896

t5 L'ark, Roswell: System of Surgery.

46 Starr, M. Allen: Brain Surgery.

\section{CHRONIC CATARRHAL GASTRITIS.}

A Clinical lecture delivered in Rush Medical College.

BY HENRY M. LYMAN, A.M., M.D.

PROFESSOR OF THE PRINCIPLES AND PRACTICE OF MEDYCINE. CHICAGO, ILL.

I desire to call your attention, this morning, to a number of patients who belong to a large class that will confront you during the whole of your active career as medical practitioners.

The first one is a young woman, 25 years of age, who had typhoid fever about a year ago. She had been employed as a shop girl for seven years previous to her illness, but had considered herself in good health till a month before the attack. She says that she was very ill for a period of five weeks and "took a lot of strong medicine." Instead of recovering her normal vigor she has remained thin and feeble. Her heart, lungs, kidneys, spleen, liver and reproductive organs appear to be healthy, but she complains of lack of appetite, epigastric distension, frequent eructation for several hours after eating and constipation of the bowels. She is listless, depressed in spirits, slightly dizzy when walking and easily put out of breath. There is anemia with a reduction of hemoglobin to 70 per cent. The pulse is small and rapid, 95 to 100 per minute. Pressure below the xiphoid cartilage is painful, and often there are transient pains between the ribs and under the left scapula. The contents of the stomach, removed with the gastric syphon about four hours after an ordinary meal, exhibit numerous traces of undigested flesh fiber; there is present a large quantity of glairy mucus, and the chemic examination indicates an excess of lactic and butyric acids, with scarcely a trace of free hydrochloric acid.

The next patient is a man, 36 years of age, thin and sharp featured. For years he has smoked and chewed excessively, but has not used alcoholic beverages. For several months he has been troubled with frequent eructations and sometimes with vomiting. The fluid that is thus evacuated is very copious in amount, and is as frothy as beer. Microscopic examination reveals the presence of sarcina ventriculi in large quantity, to say nothing of yeast fungi and numerous other microbes that are frequently present in the month and stomach.

The next patient is a veteran bartender. Fat and coarse-looking; his rubicund visage and blooming nose testify to the depth and vigor of his potations. His breath carries the offensive odor of chronic alcoholism; his tongue is thickly covered with a yellowishbrown coating; he is troubled with a frequent, dry, spasmodic cough. In the morning his head aches, he coughs and retches until he can expel a fow mouthfuls of thick, ropy mucus mixed with saliva that was swallowed during the night. Appetite is gone; whatever is eaten becomes an excitant of pain in the epi- gastrium; the liver is somewhat prominent below the short ribs and is tender on pressure. The intestines are distended with gas and are alternately affected with diarrhea and constipation; the urine is scanty, high-colored and loaded with urates. Examination of the gastric juice indicates serious reduction in all the special constituents of a healthy secretion, so that in this case it consists chiefly of mueus, saliva and water.

One more patient will suffice to illustrate the principal characteristics of the disease. $\mathrm{He}_{\theta}$ is a bookkeeper. about 48 years of.age; tall, thin and anxiouslooking. He formerly smoked excessively and kept late hours, but for the last three years he has been the victim of painful digestion and hypochondriac depression. He suffers after eating with flushing of the face, throbbing in the temples and a sensation as if something were loose and rolling around within the cranium. He is frequently so dizzy that he can not walk without a great effort. $\mathrm{He}$ is confident that his case is not understood by the physicians to whom he has applied for relief and that he is the victim of an incurable disease of the brain. The heart beats irregularly and sometimes violently. Many of his worst symptoms are relieved temporarily by eructation of gas or by the passage of flatus from the bowels, which, by the way, are obstinately constipated. He is often somnolent after eating, but is correspondingly wakeful at night. In cold weather he suffers much with neuralgia in the abdominal and thoracic walls, and almost every spring and fall he is inconvenienced for several weeks by an attack of hemorrhoids. These are sometimes replaced or preceded by a severe outbreak of urticaria, covering the whole body. Latterly there have been patches of eczema in the flexures of the knees and upon the lateral surfaces of the fingers. Last summer he had a severe attack of bronchitis with asthma, which continued until October, when it disappeared during the course of a hemorrhoidal bleeding.

Such, then, are some of the cases about which you will be frequently consulted. They all possess the common characteristic of chronic gastric catarrh. This disease often follows as the sequel of an imperfectly cured acute inflammation of the stomach. It is a common consequence of the various infective diseases; it is excited by errors of diet, hearty eating, imperfect mastication, excessive indulgence in alcoholic drinks, consumption of coarse and indigestible food and the habitual use of iced water, strong tea and coffee. It accompanies local diseases of the stomach, such as round ulcer and carcinoma; it is necessarily produced by all the disorders of circulation that are occasioned by obstruction in the portal vein, liver, heart, lungs and pleural cavities.

Could we look into the stomachs of these patients we should discover a variety of pathologic changes. The pyloric extremity of the organ is the seat of the principal disorder. There the mucosa is of a gray or brownish color, which in very chronic cases is sometimes of a dark slate color. Hemorrhagic extravasations are not uncommon in the connective tissue between the glandular organs. The distinction between the different kinds of cells in the glands themselves is often destroyed and the secreting cells are swelled with cloudy contents, or have undergone atrophy from pressure and imperfect nutrition. In some of the older cases, where the patient is anemic, there is general glandular atrophy, accompanied by the development of the symptoms of pernicious ane- 
mia. The deep brown or gray or slaty discoloration of the tissue is due to the accumulation of pigment deposited by the excessive extravasations and stagnations of blood in the capillary network that envelops the gastric glands. The submucous connective tissue and the muscular coat of the stomach also participate in the inflammatory process, so that their cellular elements multiply and increase the thickness of the gastric wall. The interstitial spaces are frequently loaded with small round cells and leucocytes, as if actual purulent formation were about to occur. By the increased thickening and swelling of the tissues thus produced, there is sometimes effected an actual stricture of the pylorus, leading finally to dilatation of the stomach. Occasionally, the external serous investment of the organ shares in the general disposition to inflammatory thickening, and sometimes the entire wall of the stomach becomes symmetrically hypertrophied, so as to reduce the cavity of the viscus, and to suggest the idea of a malignant change in the substance of the tissues, requiring the aid of the microscope to distinguish this chronic inflammatory hyperplasia from the growths of carcinoma. In many of the older cases of catarrh the mucous lining of the stomach presents a roughened, warty appearance that is caused by irregular development and contraction of the submucous connective tissue. In certain rare cases small cysts are formed by the occlusion of isolated glands, ducts and the accumulation of their imprisoned contents. By a sort of reversal of this process the interglandular elements occasionally multiply and protrude into the gastric cavity, forming mucous polypi, which are only important when large enough and sufficiently near to fall into the pyloric orifice of the stomach, more or less completely hindering the passage of food into the duodenum.

The recognition of a catarrhal inflammation of the stomach is seldom attended with difficulty, but it is not always easy to decide whether it is an uncomplicated affection or whether it is dependent upon some other grave disease in the thoracic viscera, in the kidneys or in the gastric wall itself. The discovery of cardiac, pulmonary, hepatic or renal disease will render it highly probable that the disorder is secondary to those causes of perverted circulation and nutrition. The existence of general hyperplastic thickening of the gastric wall may be suspected when the gastric tumor feels smooth, firm and devoid of irregularities in its shape. But in the absence of tumefaction and resistance to pressure in the epigastric region, the question will resolve itself into a threefold query: Are we dealing with a carcinoma, an ulcer, or merely a chronic catarrh of the stomach? If the patient be in early or middle life the probability of cancer is less than in advanced years. The absence of cachexia and discoloration of the skin. long duration of the malady and freedom from bloody vomit favor the diagnosis of chronic catarrh. Enlargement of the lymphatic glands in the left groin and supraclavicular space sometimes accompanies malignant disease of the stomach, but is not a constant symptom. The absence of free hydrochloric acid from the gastric juice is a very trustworthy, though not invariable, symptom in carcinoma. The presence of minute, coffee-ground particles of altered blood-clot in the gastric contents is a weighty indication in favor of cancer rather than atrophic catarrh of the stomach. In many instances it is impossible to discover before death the presence of a gastric tumor, but, in such cases, the absence of free hydrochloric acid, the existence of cachexia, lymphatic enlargement in the left groin and supraclavicular space, and, in the later stages of the disease, the occurrence of edema in the extremities-all these symptoms con. curring in an elderly person would suffice to warrant the diagnosis of a latent gastric cancer.' In a large proportion of the deaths occurring among old people, without definite symptoms beyond gradual wasting and exhaustion, the cause will be discovered in a latent carcinoma developed within the abdominal cavity.

The existence of an ulcer in the stomach should be inferred when the pain is more severe and narrowly circumscribed than in simple catarrh, especially if it be aggravated by eating. The disease is usually encountered among chlorotic and anemic young people, and when vomiting of blood takes place the hemorrhage is considerable, and large clots are thrown up instead of the "coffee-ground" sediment that appears in cases of cancerous bleeding.

No disease demands for its treatment more careful adaptation of means to individual necessities than chronic gastric catarrh. The daily life of the patient must be studied and all errors of hygiene, clothing, occupation and diet must be corrected. If the teeth are defective the aid of a dentist must be invoked, and the patient should be taught how to chew his food, for without thorough mastication easy digestion of the food is impossible. The food itself must be selected with a view to ease of solution in the stomach. For this reason it should resemble the food that is provided for a newly weaned infant. It must, therefore, consist chiefly of articles derived from the animal kingdom. The nursing child is a carnivorous animal by nature, and the convalescent invalid is in these respects on a level with an infant. The principal article of food should be milk-not boiled, but heated to the temperature of 160 degrees $F$., and drank before it is cooled, so as to secure the stimulant effect of a hot liquid. In fact, no cold drinks should be allowed, because the muscular coat of the stomach is usually, in these cases, relaxed and atonic. This lack of tone is aggravated by the introduction of cold liquids, which must, therefore, be forbidden. Occasionally you will meet with patients who do not like milk or who think that it renders them constipated. You must reassure such people by telling them, what is true, that if they will begin with a tablespoonful of milk at each meal, and will gradually increase the quantity to a pint, or more, their constipation will be cured. Only once have I failed in this way to overcome the repugnance of the patient, and that was a case of peculiar intolerance, in which the use of milk was always followed by an eruption of erythema.

The prescription of a milk diet may be supplemented with broths and soups that have been strained, cooled, skimmed free of fat and then heated again.

Ordinary vegetable food and fruits should be forbidden until recovery is complete. Rice may be allowed as a substitute for vegetables, but it must be steamed or boiled in a water-bath, so as to avoid the necessity of stirring while being cooked. In this way it can be furnished with every grain whole, separate from its fellow, yet thoroughly soft and perfectly well done. It should be eaten with salt and hot milk.

The craving for bread that is usually experienced by persons who have been placed on a restricted diet, 
should be satisfied with bread that has been sliced and dried in the oven before it is toasted. It should be eaten with a little butter, hot from the toasting-fork.

The animal food should be, at first, of the tenderest possible description. Calves' brains, sweetbreads, stewed tripe, tenderloin steak, lamb chops, chicken, game birds, the soft portion of the oyster, soft-boiled eggs, boiled ham, roast beef. These must be thoroughly masticated, or, if that be difficult, reduced to a pulp or mincemeat before they are taken into the mouth.

Many patients exhibit a desire for spices, mustard and vinegar, but this is a morbid craving which should not be gratified. Alcoholic beverages are forbidden because of the local irritation and the hindrance of digestion that follows the contact of alcohol with the inflamed mucosa.

Having thus regulated the diet of the patient, in many cases it is only necessary to await the favorable result. You must not be impatient at the delay which often attends recovery. Months and months of favorable conditions are frequently needful to effect a cure. Not infrequently, however, it will be desirable to have recourse to other methods beside hygiene and diet. You should then follow the indications derived from a chemical analysis of the gastric juice. I will not delay you with a review of the tests by which you may ascertain the presence, or the absence, of free acids in the stomach, for you have already become familiar with the use of the gastric syphon; and the significance of the change in color of congo paper dipped into the filtered liquid thus obtained from the stomach is something of which I need not remind you. You have often seen the behavior of the phloroglucin-vanillin reagent when heated with gastric juice; and you are well-informed regarding these simple qualitative tests. A quantitative analysis requires more time and labor; but, fortunately, it is seldom necessary outside of the laboratory of a professional chemist or physiologist. Few busy physicians can afford the time for such an examination, and in our large cities, whenever occasion requires, an exact analysis can be easily obtained from such sources. For all clinical purposes the character of the qualitative reactions is usually sufficient as a guide to treatment.

If, then, you find that the filtered juice turns red congo paper blue, you may know that it contains free acid. If now, on heating the same juice with a few drops of the phloroglucin-vanillin reagent upon a white dish, no carmin color appear, you will have learned that free hydrochloric acid is absent and that the acidity of the liquid is due to the presence of lactic, acetic, or butyric acids, for which the, appropriate tests may be employed. With a little practice you will be able to draw tolerably accurate inference regarding the relative quantity of free hydrochloric acid; but if absolute accuracy is desired you must resort to the laboratory method of qualitative analysis, as described in your handbooks of physiologic chemistry

In cases that are marked by the absence or deficiency of free hydrochloric acid, you may administer dilute hydrochloric acid. This should be given in doses of ten drops in half a glass of water with each meal. Many physicians prescribe it in larger doses, but a little reflection will show that it is not to be regarded as a substitute for the normal gastric acid. Were such the fact, it should be given in a dose of one hundred drops, diluted with nearly a quart of water. Nor should the fact that it is often grateful to the patient betray one into the advocacy of large doses, for such patients generally experience a crav. ing for acids and spices to an extent that is positively injurious. It is probably through its local antiseptic action, and by its diuretic effect upon the kidneys, that the medicinal acid is useful. Since the peptic ferments are usually reduced in quantity along with the free hydrochloric acid, it is well to add ten grains of pepsin to the acid draught.

When the patient complairs of gaseous distention of the stomach, and of eructations indicative of fermentation, you should administer remedies to prevent such decomposition of the food. Half an hour before meals, when the organ is nearly, if not quite empty, give ten grains of salol, or the same quantity of pure salicylic acid, or a grain and a half of resorcin. The efficiency of these agents is somewhat increased by giving them in peppermint water, or in capsules with a drop or two of the oil of peppermint. When the eructations are acid from the presence of lactic, butyric or acetic acid, sodium bicarbonate in scruple doses may be associated with the other antiseptic remedies. If the bowels be constipated also, ten grains of calcined magnesia should be given with the sodium powder.

But if the stomach be dilated these measures will not suffice. It then becomes necessary. to wash out the stomach every morning with the aid of the gastric syphon. Water of the temperature of 90 to 100 degrees $\mathbf{F}$., to which has been added sodium bicarbonate (one or two drachms to the pint) should be run through the tube, in and out of the stomach, until it returns clear and sweet. The disinfectant remedies above mentioned may be then administered before the patient takes his first meal.

In such cases, and the majority of chronic sufferers fall into this class, there is considerable relaxation of the muscular tone of the gastric wall. The process of irrigation and disinfection of the stomach should be, therefore, followed by the administratien of bitter tonics, which arouse the contractility of the muscular fibers. Sulphate of strychnia, in doses of onetwentieth of a grain, may be given after each meal. Ten drops of tincture of nux vomica, or a half drachm of tincture of gentian, a half drachm of the fluid extract of condurango, a wineglass of the infusion of quassia, a teacupful of the infusion of camomile flowers, or the same quantity of the infusion of eupatorium perfoliatum, are useful tonic bitters. The compound tincture of cinchona and the various elixirs of calisaya bark are often prescribed with good effect: but, as a general rule all prepartions that contain alcohol should be avoided, on account of the narcotic effect of alcohol upon the secreting glands of the mucous membrane-an effect that favors the occurrence and persistence of mucous inflammation.

For the invigoration of the general nervous apparatus of the body, electricity may be employed with great advantage. General electrization with the aid of the static machine for fifteen minutes every day is useful. Or the faradic current may be applied for the same length of time daily, with one electrode upon the back of the neck, while the other is moved slowly in every direction over the epigastrium and abdominal surface. Massage given daily is another useful method for patients who are confined to the house. Daily sponge baths with cold water are indispensable 
for the confirmation of nervous vigor. You must carefully instruct your patients with regard to these methods, together with exercise in the open air, for without such aid it is useless to expect a cure in chronic cases of exhaustion arising from gastric catarrh. It is also very important to procure for the patient a complete change of scene and occupation, for such people are usually much depressed and hypochondriacal. Hence the great benefit that may be derived from travel or from almost any new and interesting mode of bodily exercise.

When pain in the epigastric region is an important symptom, it sometimes becomes necessary to have recourse to various narcotics. Of these the most popular is alcohol, but, though it gives temporary relief, it always aggravates the inflammatory condition of the mucous membrane. A similar objection lies against the use of nitrate of silver, compound tincture of iodin, salts of bismuth, etc.; they must be administered with great caution and in small doses, lest they do more harm than good. Cocain, in doses of onetenth of a grain, often affords relief, but it is a dangerous drug and the cocain habit may be thus easily acquired. Sometimes it is actually necessary to prescribe an opiate, and in such cases it is well to exhibit small doses of Dover's powder, since the ipecacuanha which it contains is a valuable stimulant to the glands of the digestive mucosa, and does in some measure obviate the tendency to constipation that is occasioned by opiates alone. For the same reason small doses of belladonna are often useful, either alone or associated with opium or morphin, but if there is constipation codein should be given the preference, since it interferes less than other opiates with intestinal persistal. sis. Chloral hydrate may be given with advantage, in doses of five to ten grains, whenever required by a recurrence of pain, if there be any objection to opiated anodynes. The bowels should never be permitted to remain constipated. As laxatives, may be recommended the popular pills containing aloin, belladonna and strychnin, or the compound liquorice powder of the German pharmacopeia, or calcined magnesia, or the elixir of rhubarb and magnesia, or cascara cordial. If the stools exhibit a deficiency of bile, podophyllin may be given in small doses (one-tenth to one-fourth gr.) after meals, in connection with the alkalin laxatives that neutralize gastric hyperacidity.

Arthritic patients are often benefited by laxatives that contain sulphur, such as Squair's Compound Sulphur Tablets, or Garrod's Tablets, or the old-fashioned mixture of sulphur and cream of tartar. So great is the number of elegant laxative preparations thet everyone can have his own favorite, and it may suffice to point out the importance of giving the preference to those drugs which stimulate secretion and aid gastro-enteric peristalsis - in other words, the gently stimulating laxatives rather than the drastic purgatives. When constipation is chronic and the liver is engorged with blood and bile, a course of mineral waters is often beneficial. They should be drank in quantity sufficient to produce gentle catharsis, and may be taken preferably from half an hour to an hour before breakfast. As a general rule, hot mineral waters are better than those that are cold. When the natural spring water is cold, or when bottled waters are used, the morning draught should be followed by a cup of hot water, to raise the temperature of the stomach before eating, It is always best to drink the waters at their native source, in order to experience the good effects of rest, change of environment, ete., which are associated with a vacation at the springs; but when such advantages are impossible, mnch good can be obtained from the bottled waters that can be drank at home. In cases of simple gastric catarrh, the waters that contain a predominating quantity of magnesian salts, or chlorid of sodium, or of carbonate of sodium, are sufficient. When the tongue is thickly coated and yellow, indicating hepatic disorder, the waters that are richly charged with sulphate of sodium are most efficacious. The waters of Saratoga, of the deep artesian wells in eastern Michigan, when largely diluted, and the hot saline springs in Colorado and the other mountainous regions of the continent are all valuable. Of the ordinary bottled waters the Saratoga, Hunyadi .Janos, Friedrichshall, Racoczy and Rubinat waters are extensively used. For arthritic subjects the sulphurous waters may be recommended. Of these the springs at Richfield or among the Alleghany mountains and in Kentucky and Tennessee, are among the most noted. The hot springs of Arkansas possess little or no value beyond the quality of heat which they derive from the rocks through which they Hlow.

When prescribing the use of mineral waters or their artificial substitutes - solutions of magnesian and sodic salts - care should be taken to caution the patient against their too persistent use. Few can drink the stronger waters longer than five or six weeks without exciting an inflammation of the gastrointestinal mucosa that may be very difficult to heal. It is better to use them for a month or six weeks at the springs and then to abstain for a number of weeks, or to have recourse, if needed, to the mild vegetable laxatives. It is always important in this, as in all other protracted courses of medication, to provide for occasional intermissions, in order to prevent both local irritation and the establishment of tolerance of the drug that is employed.

\section{ANTITOXIN AND DIPHTHERIA.}

Read before the Harrisburg Academy of Medicine, Dec. $29,1896$.

\section{BY W. H. SEIBERT, M.D.}

STEELTON, PA.

At a meeting held here about a year ago I read a paper entitled "Diphtheria," in which the subject was treated in its entirety and some reference was made to the use of antitoxin serum.

The paper which I present tonight deals chiefly with antitoxin and some reference is made to diphtheria as seems to be proper or relevant to the subject.

Careful observations were made during this year in the treatment of all cases occurring in my practice, and the results will be presented briefly, together with statistics of our town so far as applies to results in the treatment with and without the serum. In addition, there will be presented statistics of many of the largest cities of the United States relating to frequency of the disease, comparative mortality, mortality rates of the pre-antitoxin years as compared with the mortality of the present year.

In referring to the history of diphtheria it may be said that it comprises three main periods, the first of which dates from the earliest times of medical history to the sixteenth century. In this period various descriptions are given of the different epidemics of the disease by Hippocrates, Aretæus, Galen and various other writers. The second period extends from 